\title{
Effet de traitements alimentaires de légumes et de céréales sur la radioactivité des produits transformés
}

\author{
S. ROUSSEL-DEBET*, J. REAL*
}

(Manuscrit reçu le 18 juillet 1994,

révisé le 24 décembre 1994, accepté le 29 janvier 1995)

RÉSUMÉ La diminution de radioactivité de ${ }^{134} \mathrm{Cs},{ }^{85} \mathrm{sr},{ }^{106} \mathrm{Ru},{ }^{57} \mathrm{Co},{ }^{110 \mathrm{~m}} \mathrm{Ag}$ au cours de traitements alimentaires de légumes et de céréales a été mesurée. Les opérations de lavage et de blanchiment de légumes contaminés directement par un dépôt unique d'aérosols induisent une réduction de l'activité massique (variable selon le végétal, le radionucléide et le stade végétatif du végétal au moment du dépôt), qui peut atteindre $90 \%$ pour le césium. L'efficacité de ces traitements est moindre sur des légumes contaminés par voie racinaire, à l'exception du parage des légumes racines. La mise en conserve après parage et blanchiment de légumes contaminés en césium, strontium, cobalt et ruthénium par transfert indirect s'avère être assez efficace, avec une radioactivité résiduelle allant de $\mathbf{3 0}$ à $50 \%$ pour le haricot vert et de 5 à $20 \%$ pour la carotte. Des mesures d'isotopes stables du césium et du strontium effectuées sur des céréales avant et après traitements industriels mettent en évidence une diminution importante de l'activité massique des produits transformés ; les facteurs de transformation sont de 0,1 à 0,2 pour la transformation du blé en farine et de 0,1 à 0,4 pour le blanchiment du riz.

ABSTRACT The decrease of radioactivity for ${ }^{134} \mathrm{Cs},{ }^{85} \mathrm{Sr},{ }^{106} \mathrm{Ru},{ }^{57} \mathrm{Co},{ }^{110} \mathrm{~m} \mathrm{Ag}$ during food processing was measured. Washing and bleaching vegetables directly contaminated by a single aerosol deposit led to a radioactivity decrease (depending on the vegetable, the radionuclide and the time of deposition) up to $90 \%$ for caesium. The efficiency of these processes was less important when the vegetables were contaminated by root transfer, except for peeling of root vegetables. Canning of vegetables undirectly contamined by caesium, strontium, cobalt and ruthenium, after paring and bleaching, was rather interesting with residual radioactivity varying from 30 to $50 \%$ for green beans and from 5 to $20 \%$ for carrots. Measurements of stable caesium and strontium in cereals before and after industrial processing showed an important activity decrease; retention factors varied from 0.1 to 0.2 for wheat milling and 0.1 to 0.4 for rice processing.

* Institut de protection et de sûreté nucléaire, Département de protection de l'environnement et des installations, Service d'études et de recherches sur les transferts dans l'environnement, IPSN/CEA, 13108 St Paul-lez-Durance Cedex. 


\section{Introduction}

Des études destinées à quantifier la diminution de l'activité massique de végétaux lors de traitements intervenant après la récolte ont été effectuées. L'objectif est de présenter sous forme synthétique les résultats issus de plusieurs expérimentations afin qu'ils puissent être utilisés dans les modèles de transferts. Les travaux présentés concernent :

- d'une part l'évolution des activités du césium, strontium, ruthénium, cobalt, argent lors de préparations alimentaires de légumes contaminés en laboratoire par différentes techniques et, en particulier, par dépôts d'aérosols ; les opérations étudiées sont le parage, qui consiste à éliminer les parties non consommables, le lavage, le blanchiment, qui est une brève cuisson à l'eau, ainsi que la conservation par appertisation, c'est-à-dire en boîtes fermées et stérilisées ;

- d'autre part, des résultats relatifs au cobalt, césium et strontium (mesurés à partir des isotopes stables), obtenus sur des céréales prélevées dans l'industrie au cours d'opérations de meunerie et de semoulerie.

\section{Méthodes}

\section{Parage, lavage et blanchiment de légumes contaminés à divers stades végétatifs, par dépôt unique d'aérosols sur la partie aérienne des végétaux}

Des salades, haricots verts, tomates, pommes de terre, carottes, radis sont soumis à un dépôt unique de radionucléides sous forme d'aérosols secs ou humides. Les aérosols humides sont produits par vaporisation de radionucléides en solution aqueuse. Les aérosols secs sont fabriqués à partir d'une poudre composée d'éléments stables et de radionucléides, supposés représenter un mélange émis en cas d'accident de réacteur. Ce mélange est placé dans un four dont la montée en température $\left(2850^{\circ} \mathrm{C}\right)$ permet l'émission de particules radioactives dont le diamètre moyen est de l'ordre de $1 \mu \mathrm{m}$. Au moment du dépôt, les végétaux sont à différents stades de développement : maturité, mimaturité, plantule, semis. Les radionucléides étudiés sont l'argent $110 \mathrm{~m}$ sous forme métal, le césium 134 sous forme chlorure ou carbonate, le ruthénium 106 sous forme hydroxyde et le strontium 85 sous forme carbonate.

Après la contamination, les cultures sont poursuivies jusqu'à maturité des parties consommables, dans un délai variant de un jour à plusieurs mois, selon le stade végétatif à la contamination. Ensuite, les végétaux sont récoltés et subissent' des préparations culinaires simples, afin d'évaluer l'importance de la diminution de radioactivité qui en résulte. Comme l'indique le tableau I, les parties comestibles des légumes sont parées et lavées, puis subissent un blanchiment durant quelques minutes dans l'eau bouillante.

Les résidus de parage, les eaux de lavage et de cuisson ainsi que les végétaux préparés sont ensuite broyés et mélangés à un gel qui assure une bonne homogénéisation de chaque échantillon [6]. Les mesures sont faites par spectrométrie gamma Ge. 
TABLEAU I

Résumé des expérimentations effectuées concernant le parage (P), le lavage (L) et le blanchiment $(B)$, suite à un dépôt d'aérosols sur divers végétaux

Summary of the experiments relating to peeling ( $P$ ), washing (L) and bleaching (B) following aerosol deposition on various vegetables

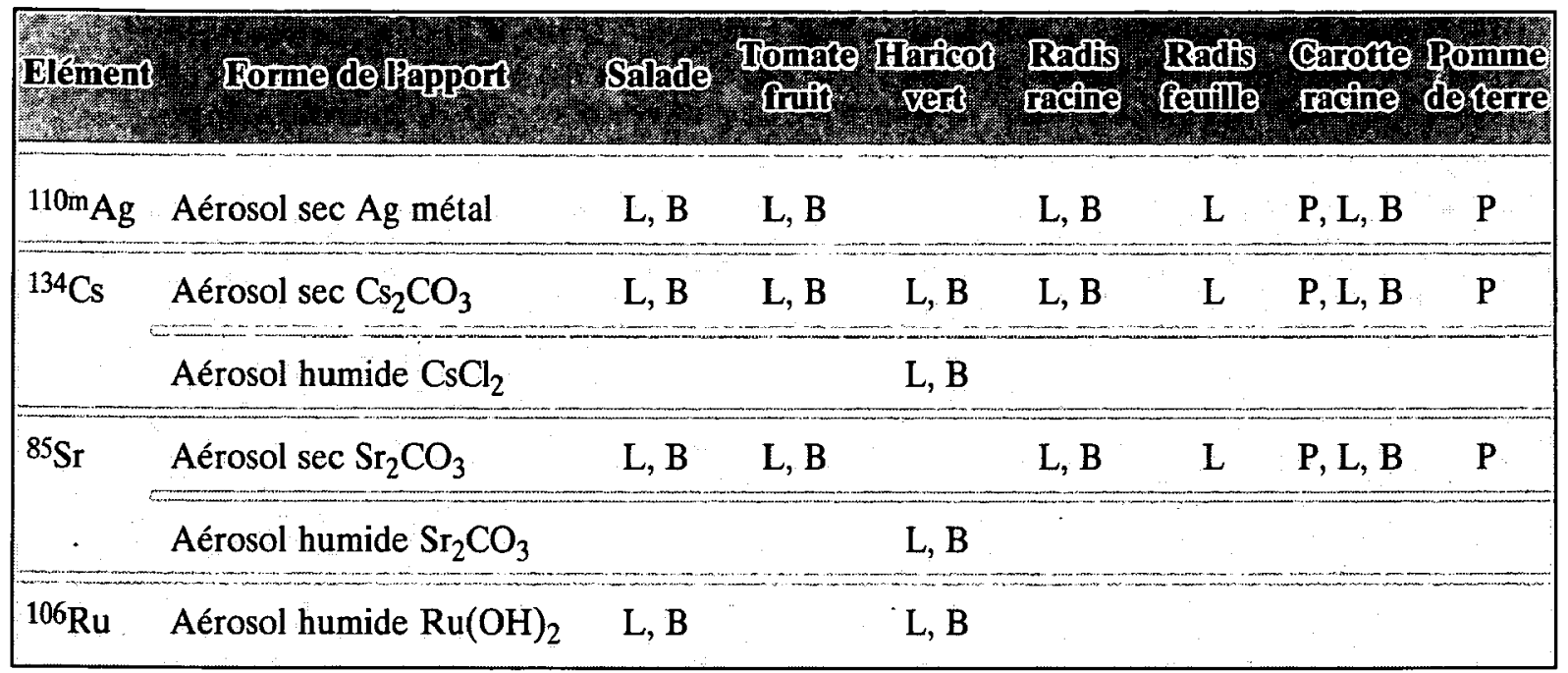

\section{Parage, lavage, et blanchiment des légumes contaminés par d'autres techniques}

Le tableau II résume les expérimentations réalisées pour trois modes de contamination des végétaux.

Une première série d'essais $(a)$ est faite avec des végétaux contaminés par voie racinaire. Des carottes et des haricots verts sont cultivés dans des bacs de sol enrichi en ${ }^{134} \mathrm{Cs},{ }^{57} \mathrm{Co},{ }^{106} \mathrm{Ru}$ d'une part et ${ }^{85} \mathrm{Sr}$ d'autre part, sous forme de chlorures. Les radionucléides sont apportés de façon homogène avant le semis.

D'autres essais $(b)$ concernent des cultures de végétaux sur les sols ayant subi un dépôt unique d'aérosols secs $\left(\mathrm{Cs}_{2} \mathrm{CO}_{3}, \mathrm{Sr}_{2} \mathrm{CO}_{3}, \mathrm{Ag}\right.$ métal $)$, les plantes étant semées après homogénéisation de ces sols.

Une troisième série expérimentale $(c)$ est réalisée à partir de cultures effectuées sur sol non contaminé. Les végétaux sont extraits du sol à maturité puis contaminés par trempage des feuilles (carottes) ou des racines (haricots verts) durant 24 à $48 \mathrm{~h}$ dans une solution contaminée en chlorures de ${ }^{134} \mathrm{Cs},{ }^{57} \mathrm{Co}$, ${ }^{106} \mathrm{Ru},{ }^{85} \mathrm{Sr}$. Cette technique également utilisée par Cawse [3] a pour but de simplifier les modalités de contamination des végétaux, notamment pour le ruthénium qui, étant très faiblement transféré, nécessiterait des niveaux de contamination des sols incompatibles avec les normes de radioprotection.

Après la contamination, les parties comestibles récoltées sont traitées de la façon suivante : elles subissent un prélavage éventuel, destiné à éliminer la terre, puis, successivement un parage, un lavage et un blanchiment durant 


\section{TABLEAU II}

Autres modes de contamination des légumés : (a) cultures sur sol contaminé de façon homogène par des chlorures ; $(b)$ cultures sur sol homogénéisé après un dépôt unique d'aérosols secs ; (c) contamination après récolte de la plante extraite du sol, par un trempage des feuilles (carotte) ou des racines (haricot)

Other contamination methods : (a) cultivation on a soil homogeneously contaminated by chlorides; (b) cultivation on a soil homogenized after a single surface deposit of dry aerosols ; (c) contamination, after gathering, by soaking of leaves (carrots) or roots (beans)

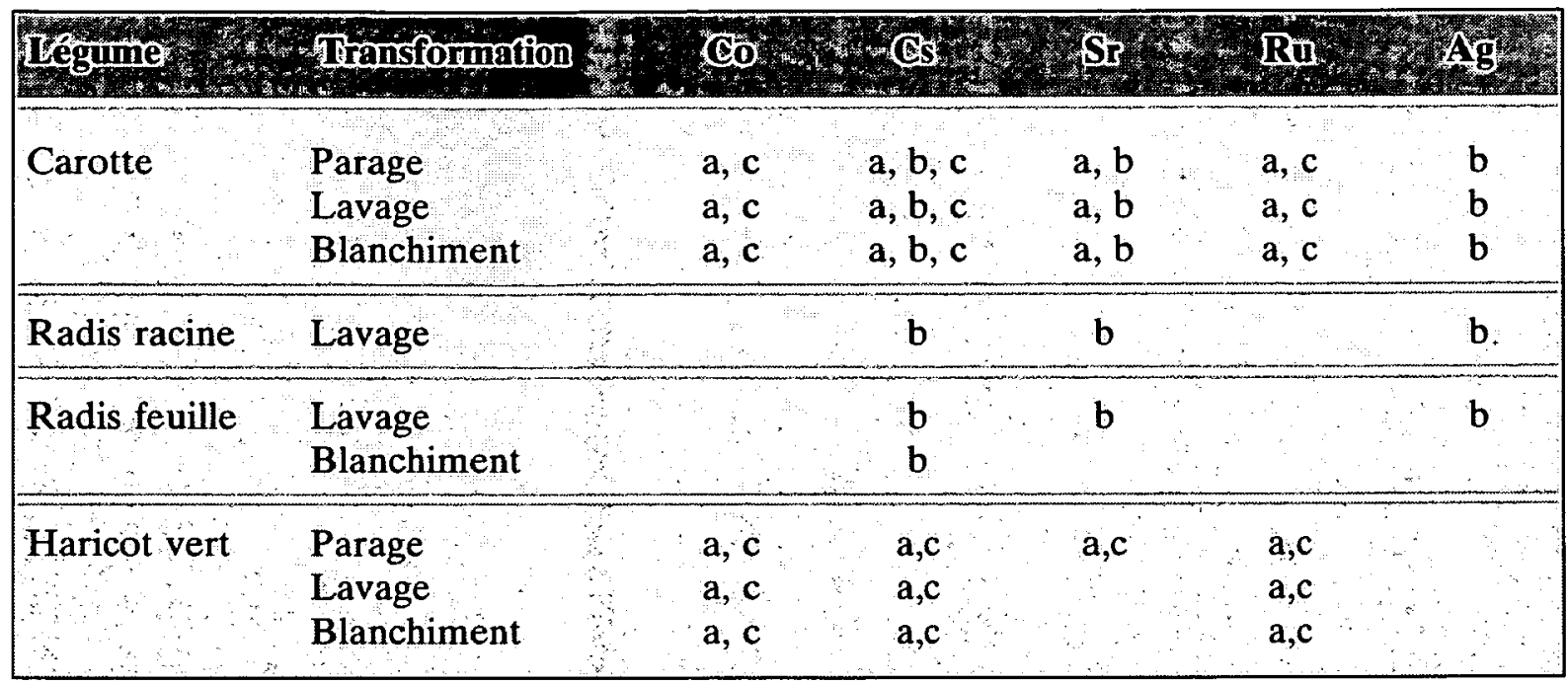

5 min à l'eau bouillante. Les fractions (résidus de parage, eaux de lavage, de blanchiment) sont conditionnées par minéralisation en phase nitrique ou par broyage avec un gel puis mesurées par spectrométrie gamma Ge.

\section{Mise en conserve de légumes (Fig. 1)}

Les carottes et des haricots verts contaminés soit par voie racinaire $(a)$, soit par trempage dans une solution contaminée $(c)$, sont prélavés, parés et blanchis. Ils sont ensuite mis en boîtes de conserve métalliques avec de l'eau salée à $2 \%$. Les boîtes sont serties et stérilisées puis stockées durant un mois. Après l'ouverture des boîtes, le végétal séparé du jus de conserve est égoutté et rincé. Les fractions sont minéralisées en phase nitrique puis mesurées par spectrométrie gamma Ge (Fig. 1).

\section{Etude des traitements industriels de céréales}

Des prélèvements de grains, de farine, de semoule de blé ainsi que de riz avant et après décorticage sont faits en usine. Le but est de quantifier les pertes d'éléments stables lors des traitements industriels qui sont difficilement reproductibles en laboratoire. Pour assurer une bonne détection, les échantillons sont de l'ordre de $2,5 \mathrm{~kg}$ sec. Ils sont calcinés, puis minéralisés en phase 


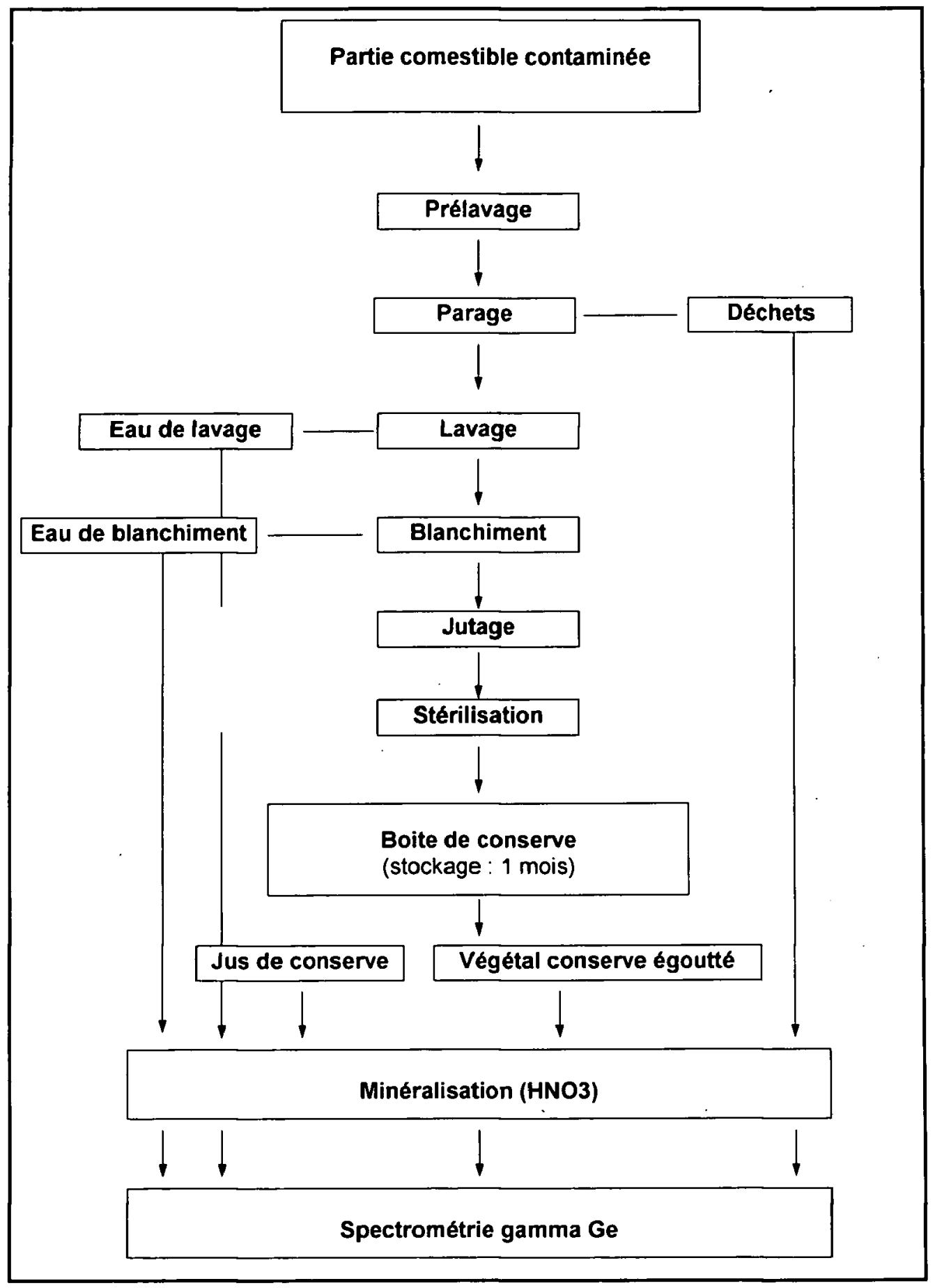

Fig. 1 - Schéma du mode opératoire pour la réalisation expérimentale de conserves de légumes.

Schematic diagram showing the experimental canning of vegetables.

nitrique et analysés par spectrométrie de masse (ICP/MS : "induced coupled plasma mass spectrometry"). Les teneurs en éléments stables des céréales avant usinage sont indiquées au tableau III. On admet que les isotopes stables et radioactifs ont le même comportement. 
TABLEAU III

Concentrations en éléments stables des grains de céréales avant usinage ( $\mu \mathrm{g} / \mathrm{kg}$ frais)

Concentrations of stable elements in cereal grains before industrial processing ( $\mu \mathrm{g} / \mathrm{kg}$ fresh)

\begin{tabular}{|lcccc|}
\hline & Sr & Co & Cs \\
\hline Ble dur & 1450 & 2,7 & 4,0 \\
\hline Blé tendre & 1440 & 9,6 & 4,9 \\
\hline Riz long & 1280 & 9,5 & 1,3 \\
\hline Riz rond & 1335 & 11,6 & 1,8 \\
\hline
\end{tabular}

\section{Résultats et discussion}

\section{Effet du parage, du lavage et du blanchiment des végétaux contaminés par aérosols}

Les tableaux IV à VI, présentent les résultats relatifs aux légumes contaminés par aérosols. Ces tableaux résument la répartition de l'activité (en pourcentage moyen par rapport au légume brut) dans les résidus de parage, les eaux de lavage et de blanchiment, ainsi que dans le légume après traitement.

En ce qui concerne le parage, le tableau IV permet d'examiner les traitements des légumes "racines" (dont la partie comestible est souterraine). Le pelage de pommes de terre contaminées au stade maturité conduit à une décontamination efficace : il reste dans la pulpe de 5 à $15 \%$ de l'activité initiale, alors que pour une contamination au stade plant, il reste 30 à $50 \%$ de l'activité totale. L'épluchage de carottes contaminées au stade plant élimine 60 à $70 \%$ de l'activité, pour une perte de masse de l'ordre de $50 \%$. Le lavage et le blanchiment n'ont qu'un effet mineur par rapport au parage.

Les effets du lavage et du blanchiment dépendent de l'importance des surfaces directement soumises au dépôt qui varie avec le stade végétatif au moment de la contamination et le type de légume.

Dans le cas de la salade, les valeurs présentées tableau V montrent l'absence de corrélation significative entre le stade végétatif au moment du dépôt et l'importance de la contamination résiduelle après traitement. Le lavage permet une diminution d'activité de $10 \%$ pour l'argent, de 40 à $50 \%$ pour le ruthénium, d'environ $60 \%$ pour le strontium et de 10 à $40 \%$ pour le césium. L'effet d'un blanchiment après lavage est très faible pour l'argent. En, revanche, pour le strontium et le ruthénium, il permet d'enlever 20 à $30 \%$ de l'activité initiale. Pour le césium, la diminution supplémentaire d'activité est supérieure à $50 \%$. 


\section{TABLEAU IV}

Répartition de l'activité, en pourcent de l'activité initiale du légume brut, lors des traitements de légumes "racines" contaminés par un dépôt d'aérosols Activity distribution during the processing of root vegetables following aerosol deposition (in percent of the initial activity of the raw vegetable)

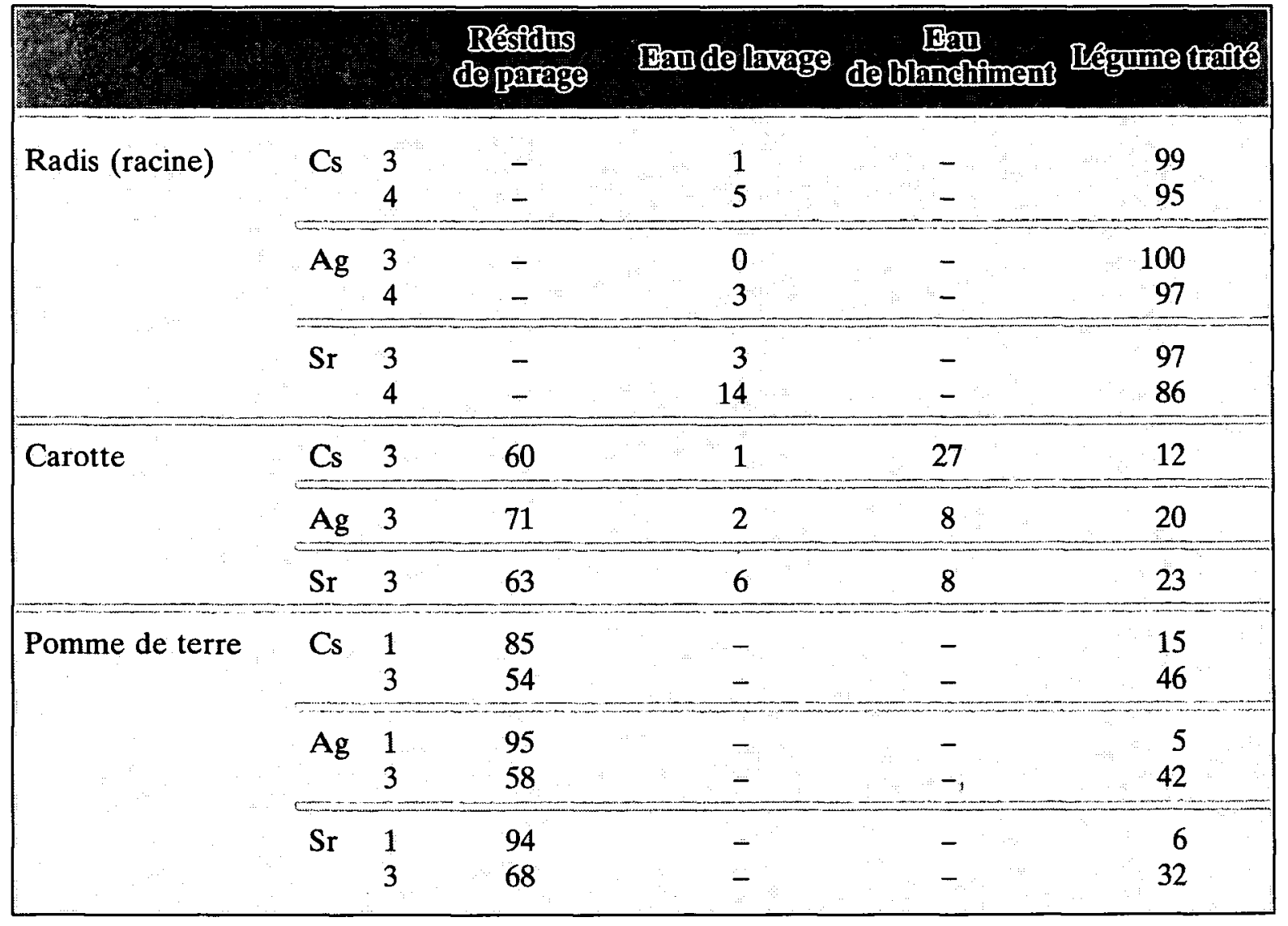

Stade du végétal à la contamination $: 1=$ maturité $; 3=$ plant $; 4=$ semis.

L'efficacité globale des traitements semble augmenter avec la solubilité du radionucléide : l'activité éliminée est de 15 à $30 \%$ pour l'argent qui est strictement insoluble et de l'ordre de $90 \%$ pour le césium (totalement soluble). Pour le ruthénium et pour le strontium, la solubilité est partielle et les valeurs obtenues sont intermédiaires.

En ce qui concerne la tomate (tableau VI), l'effet du lavage et du blanchiment dépend fortement du stade végétatif au moment de la contamination. Le lavage est particulièrement efficace pour un fruit contaminé à maturité : l'activité résiduelle est de 10 à $30 \%$, alors que pour une contamination au stade plant on s'approche des valeurs relatives à un transfert indirect. Le blanchiment enlève encore 10 à $20 \%$ de l'activité du végétal lavé.

Pour le haricot, l'effet du lavage dépend peu du stade végétatif au moment du dépôt pour le strontium et le césium, avec une contamination résiduelle de $50 \%$ en strondium et de $90 \%$ en césium. Le blanchiment accroît l'élimination 


\section{TABLEAU V}

Répartition de l'activité, en pourcent de l'activité initiale du légume brut, lors des traitements (lavage et blanchiment) de légumes "feuilles" contaminés par un dépôt d'aérosols

Activity distribution during the processing (washing and bleaching) of leafy vegetables following aerosol deposition (in percent of the initial activity of the raw vegetable)

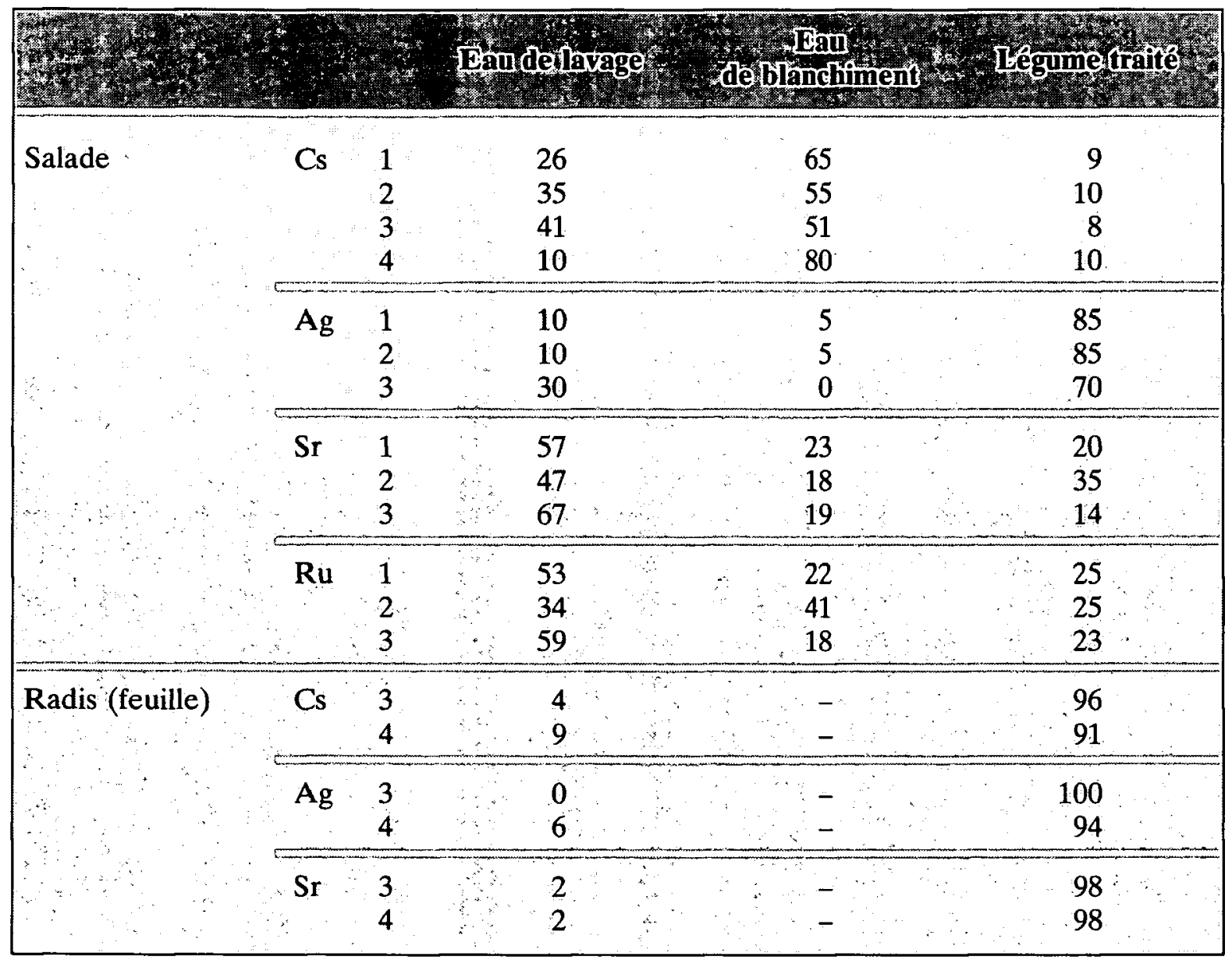

Stade du végétal à la contamination $: 1=$ maturité $; 2=$ mi-maturité, $3=$ plant $; 4=$ semis.

d'activité et, pour le lavage suivi d'un blanchiment, l'activité résiduelle est de l'ordre de $40 \%$ pour le strontium, de 10 à $40 \%$ pour le ruthénium, et de 40 à $50 \%$ pour le césium.

\section{Effet du parage, du lavage et du blanchiment de végétaux pour les autres modes de contaminations}

Le tableau VII présente les valeurs moyennes de l'activité éliminée dans les eaux de lavage et de blanchiment, en pourcent de l'activité initiale du légume brut, pour des végétaux contaminés par transfert racinaire ou par trempage en solution contaminée. On observe, pour chaque type de légumes, une relative homogénéité des résultats obtenus quels que soient le mode de contamination et le radionucléide. 
TABLEAU VI

Répartition de l'activité, en pourcent de l'activité initiale du légume brut, lors des traitements (lavage, blanchiment) de tomates et de haricots verts contaminés par un dépôt d'aérosols

Activity distribution during the processing (washing and bleaching) of tomatoes and green beans following aerosol deposition

(in percent of the initial activity of the raw vegetable)

\begin{tabular}{|c|c|c|c|c|c|c|}
\hline 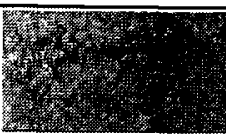 & & 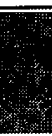 & $\begin{array}{l}\text { Eau } \\
\text { Lava }\end{array}$ & $\begin{array}{l}\text { Eau } \\
\text { nch }\end{array}$ & & 8 \\
\hline Tomate & Cs & $\begin{array}{l}1 \\
3\end{array}$ & $\begin{array}{r}90 \\
5\end{array}$ & $\begin{array}{r}0 \\
25\end{array}$ & $\begin{array}{l}10 \\
70\end{array}$ & $\begin{array}{r}5 \\
20 \\
\end{array}$ \\
\hline & $\mathrm{Ag}$ & $\begin{array}{l}1 \\
3\end{array}$ & $\begin{array}{l}70 \\
15\end{array}$ & $\begin{array}{r}5 \\
15\end{array}$ & $\begin{array}{l}25 \\
70\end{array}$ & $\begin{array}{r}5 \\
20\end{array}$ \\
\hline & $\mathrm{Sr}$ & $\begin{array}{l}1 \\
3\end{array}$ & $\begin{array}{l}80 \\
15\end{array}$ & $\begin{array}{r}5 \\
25\end{array}$ & $\begin{array}{l}15 \\
60\end{array}$ & $\begin{array}{r}5 \\
20\end{array}$ \\
\hline Haricot vert & $\mathrm{Cs}$ & $\frac{1}{2}$ & $\begin{array}{r}14 \\
2\end{array}$ & $\begin{array}{l}60 \\
44\end{array}$ & $\begin{array}{l}26 \\
52\end{array}$ & - \\
\hline & $\mathrm{Sr}$ & $\begin{array}{l}1 \\
2\end{array}$ & $\begin{array}{l}43 \\
46 \\
\end{array}$ & $\begin{array}{r}6 \\
18 \\
\end{array}$ & $\begin{array}{l}51 \\
36\end{array}$ & - \\
\hline & $\mathbf{R u}$ & $\begin{array}{l}1 \\
2\end{array}$ & $\begin{array}{l}33 \\
76\end{array}$ & $\begin{array}{l}28 \\
14\end{array}$ & $\begin{array}{l}39 \\
10\end{array}$ & - \\
\hline
\end{tabular}

Stade du végétal à la contamination : $1=$ maturité $; 2=$ mi-maturité, $3=$ plant.

L'épluchage des légumes de type racine élimine de 60 à $90 \%$ de l'activité initiale, y compris pour les légumes contaminés à maturité par trempage des feuilles, et pour lesquels il n'y a pas de contact entre le sol contaminé et la racine. La perte de masse varie entre $30 \%$ et $60 \%$, les facteurs de transformation (rapport des activités massiques des légumes après et avant traitement) sont donc de l'ordre de 0,5. Pour les gousses (haricots), l'efficacité du parage est minime. L'épluchage élimine 10 à $30 \%$ de l'activité initiale, pour une perte de masse de l'ordre de $10 \%$. Dans ce cas, le facteur de transformation est proche de l'unité.

Le lavage (précédé d'un prélavage et d'un pelage pour les végétaux racines) n'a pas, en général, d'effet significatif sur les activités massiques des radionucléides étudiés (l'activité éliminée est en moyenne inférieure à $4 \%$, attribuable à la fixation des radionucléides sur les récipients), ce qui est logique puisqu'il n'y a pas de contamination sur les surfaces externes des parties consommables.

Le blanchiment enlève de 5 à $20 \%$ de l'activité, quels que soient les radionucléides et quelle que soit la forme de l'apport. 
TABLEAU VII

Répartition de l'activité, en pourcent de l'activité initiale du légume brut, lors des traitements (parage, lavage, blanchiment) de légumes contaminés par transfert indirect

Activity distribution during the processing (peeling, washing and bleaching) of vegetables following undirect uptake

(in percent of the initial activity of the raw vegetable)

\begin{tabular}{|c|c|c|c|c|c|c|}
\hline rist & 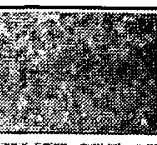 & 4 & ésid & Eav & Each & rgit \\
\hline Co & Racine & a & 66 & 1 & 15 & 18 \\
\hline & & c & 74 & 1 & 5 & 20 \\
\hline & Fnuit & $a$ & 10 & 06 & 9 & 80 \\
\hline & & $\begin{array}{l}\mathrm{a} \\
\mathrm{c}\end{array}$ & $\begin{array}{c}10 \\
5\end{array}$ & $\begin{array}{l}0,0 \\
1\end{array}$ & 8 & 86 \\
\hline Cs & Racine & a & 79 & 1 & 8 & 12 \\
\hline & & b & 76 & 0,3 & 20 & 4 \\
\hline & & c & 78 & 1 & 10 & 11 \\
\hline & Feuille & $\mathrm{b}$ & - & 15 & 80 & 5 \\
\hline & Fruit & $a$ & 15 & 0,2 & 14 & 71 \\
\hline & & $\mathrm{b}$ & 5 & 1 & 7 & 87 \\
\hline Sr & Racine & $\mathrm{a}$ & 94 & 0,3 & 5 & 1 \\
\hline & & $\mathbf{b}$ & 64 & 6 & 20 & 10 \\
\hline & Feuille & $\mathbf{b}$ & - & 1 & - & 99 \\
\hline & Fruit & $\mathbf{a}$ & 34 & 0,3 & 5 & 61 \\
\hline & & c & 30 & 0,1 & 5 & 65 \\
\hline$R u$ & Racine & $\mathbf{a}$ & 53 & 0,2 & 17 & 30 \\
\hline & & c & 79 & 0,1 & 3 & 18 \\
\hline & Fruit & $\mathbf{a}$ & 17 & 0,6 & 11 & 71 \\
\hline & & c & 16 & 0,1 & 6 & 78 \\
\hline $\mathrm{Ag}$ & Racine & $\mathbf{b}$ & 81 & 3 & 15 & 1 \\
\hline & Feuille & b & - & 2 & - & 98 \\
\hline
\end{tabular}

a : cultures sur sol contaminé de façon homogène par des chlorures; b : cultures sur sol homogénéisé après un dépôt unique d'aérosols secs ; $\mathbf{c}$ : cultures contaminées par un trempage des feuilles (haricot) ou des racines (carottes), après récolte; racine : légume racine (carotte, radis), feuille: légume feuille (salade, feuilles de radis), gousse: légume gousse (haricot vert).

Le cas du césium pour les légumes feuilles est particulier. Le lavage et le blanchiment éliminent respectivement $15 \%$ et près de $85 \%$ de l'activité initiale, pour un végétal cultivé sur un sol homogénéisé après un dépôt d'aérosols. L'absence d'éléments de comparaison avec les autres modes de contamination ne permet pas de savoir s'il s'agit d'une règle générale. 


\section{Conserves de légumes}

De façon générale, la conservation par appertisation est un procédé d'efficacité reconnue pour diminuer l'activité des légumes [3]. Ceci est confirmé par les résultats présentés au tableau VIII qui donne les valeurs des facteurs de transformation $F_{\mathrm{tf}}(\mathrm{Bq} / \mathrm{kg}$ frais de produit final par $\mathrm{Bq} / \mathrm{kg}$ frais de produit initial), et les rendements en masse, $R_{\mathrm{f}}(\mathrm{kg}$ frais de produit final par $\mathrm{kg}$ frais de produit initial) entre les légumes bruts et les conserves appertisées.

La diminution de radioactivité est imputable, pour une partie, à l'élimination des parties non consommables lors du parage, ainsi qu'à un faible transfert des radionucléides vers l'eau de blanchiment, auxquels s'ajoute pour une part non négligeable un transfert des radionucléides vers le jus de la boîte, probablement lors de la stérilisation. Pour le haricot vert, l'activité résiduelle est de $30 \%$ pour le césium, et de l'ordre de 40 à $50 \%$ pour le strontium, le cobalt et le ruthénium, avec une diminution de masse négligeable. Dans la conserve de carotte, la majorité de l'activité est éliminée au parage (que le légume soit contaminé par voie racinaire ou foliaire). Dans la conserve, il ne reste que 5 à $10 \%$ de l'activité initiale du légume brut.

TABLEAU VIII

Facteur de transformation $F_{t f}(\mathrm{~Bq} / \mathrm{kg}$ frais de produit final par $\mathrm{Bq} / \mathrm{kg}$ frais de produit initial) et rendement en masse $R_{\mathrm{f}}$ ( $\mathrm{kg}$ frais de produit final par $\mathrm{kg}$ frais de produit initial) entre le végétal brut récolté et le végétal mis en conserve appertisée après parage, lavage et blanchiment. Végétaux contaminés par transfert indirect par des radionucléides sous forme de chlorures Retention factor $F_{\mathrm{tf}}(\mathrm{Bq} / \mathrm{kg}$ fresh of the final product per $\mathrm{Bq} / \mathrm{kg}$ fresh

of the initial product) and mass yield $R_{\mathrm{f}}(\mathrm{kg}$ fresh of the final product per $\mathrm{kg}$ fresh of the initial product) between raw vegetables and canned vegetables (undirect transfert through radionuclides as chlorides)

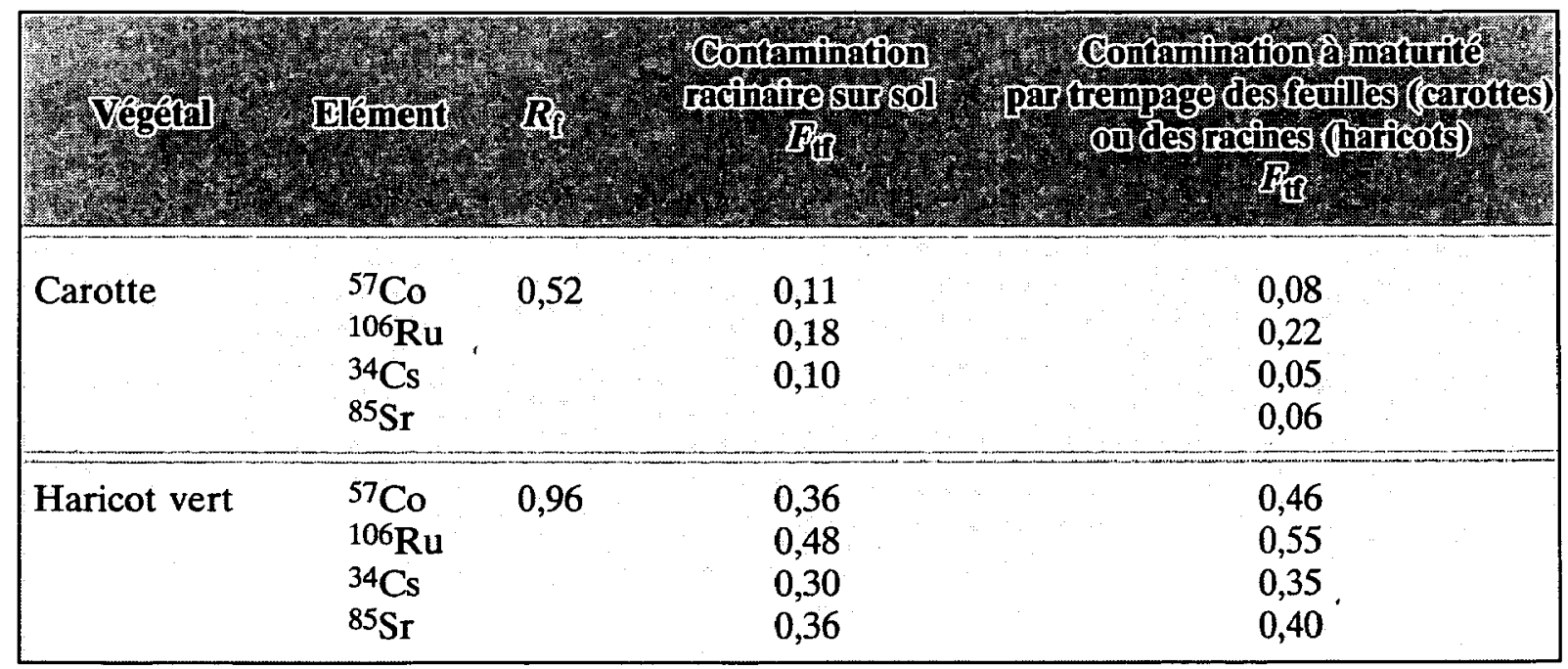




\section{Traitement industriel des céréales}

Les techniques de traitement des céréales sont pour l'essentiel des opérations physiques (abrasion, mouture, séparation par tamisage) dont l'objectif est d'éliminer les parties externes des grains. Elles aboutissent à une décontamination supérieure à une simple perte de masse car les radionucléides sont proportionnellement plus concentrés dans les zones externes du grain que dans les parties internes. Corrélativement, les sous-produits (balles, sons, farines d'abrasion) ainsi que les germes retiennent la majorité de l'activité des grains bruts.

Le tableau IX indique les valeurs des facteurs de transformation $F_{\mathrm{tf}}(\mathrm{Bq} / \mathrm{kg}$ frais de produit final par $\mathrm{Bq} / \mathrm{kg}$ frais de produit initial), déduites des mesures des isotopes stables, et les rendements en masse $R_{\mathrm{f}}(\mathrm{kg}$ frais de produit final par $\mathrm{kg}$ frais de produit initial) pour les transformations industrielles des céréales.

Le riz récolté dit "paddy" est transformé par deux opérations successives d'abrasion entre des meules. La première permet la séparation des enveloppes externes et donne le riz cargo, la seconde, qui élimine le péricarpe et le germe, fournit le riz blanchi. Ces deux processus aboutissent à une élimination importante de l'activité : il ne reste dans le riz blanchi que 10 à $30 \%$ de l'activité initiale en strontium, césium et cobalt.

TABLEAU IX

Facteur de transformation $F_{\text {tf }}(\mathrm{Bq} / \mathrm{kg}$ frais de produit final par $\mathrm{Bq} / \mathrm{kg}$ frais de produit initial) et rendement en masse $R_{\mathrm{f}}$ (kg frais de produit final par $\mathrm{kg}$ frais de produit initial) entre les céréales brutes récoltées

et les dérivés céréaliers (valeurs dérivées des mesures des isotopes stables sur des échantillons prélevés dans l'industrie)

Retention factor $F_{\mathrm{tf}}(\mathrm{Bq} / \mathrm{kg}$ fresh of the final product per $\mathrm{Bq} / \mathrm{kg}$ fresh of the initial product) and mass yield $R_{f}$ ( $\mathrm{kg}$ fresh of the final product per $\mathrm{kg}$ fresh of the initial product) between the raw grains and the processed cereals (values derived from the stable isotopes measured on industrial samples)

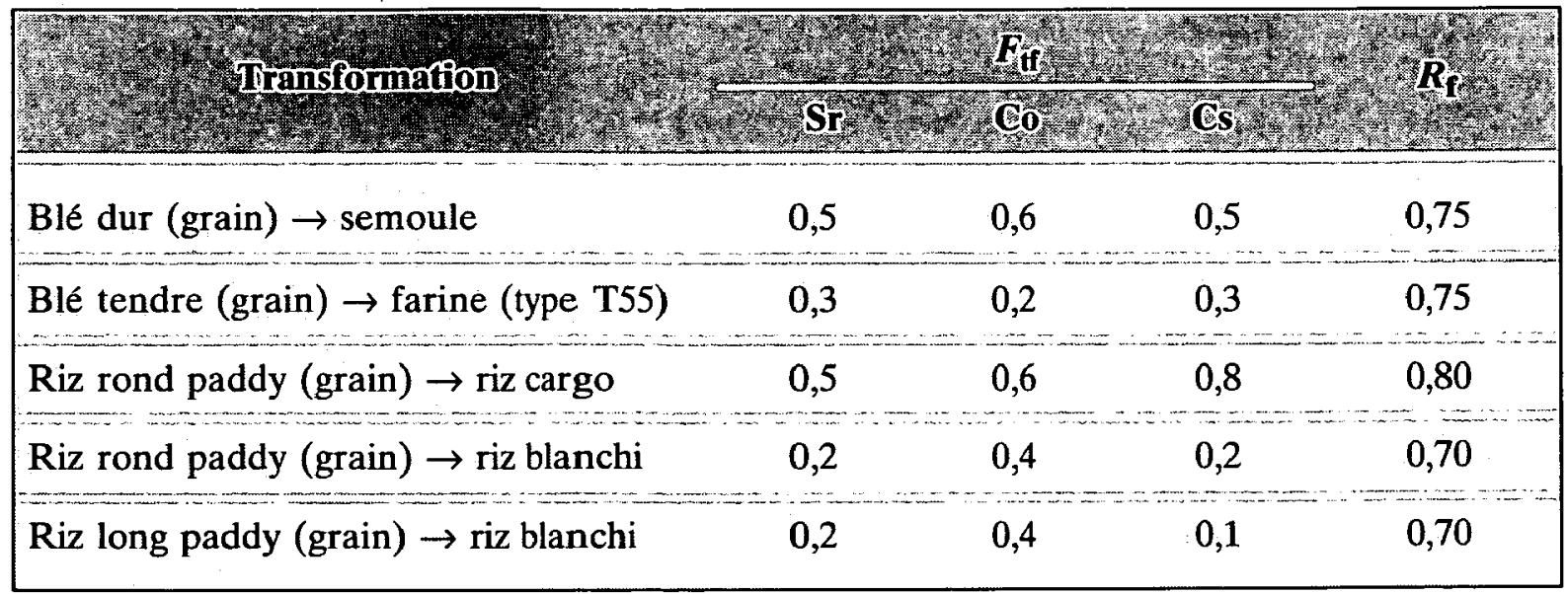


En ce qui concerne le blé, les opérations de meunerie ont pour objectif l'extraction du gluten des grains. Ces techniques se révèlent un moyen efficace d'éliminer une partie de l'activité du grain : les farines blanches retiennent 15 à $20 \%$ du césium et du strontium présents dans le grain brut. La fabrication de semoule de blé dur est un peu moins efficace : 35 à $40 \%$ du strontium, de césium, du cobalt restent dans le produit usiné. Les valeurs obtenues par les mesures du césium stable sont en accord avec celles de la littérature relatives aux mesures d'isotopes radioactifs sur des blés [1, 4-5].

\section{Conclusions}

Les procédés étudiés pour deux composants majeurs de l'alimentation d'origine végétale (légumes et céréales) sont largement pratiqués et ils permettent d'abaisser de façon notable le niveau de contamination des produits agricoles. La diminution de l'activité initiale est variable suivant l'origine de la contamination, les radionucléides, les produits et les processus.

Après une contamination par aérosols d'une production en cours de culture (maturité ou mi-maturité), on peut considérer que le lavage suivi d'un blanchiment élimine $50 \%$ à $90 \%$ du césium, $50 \%$ à $80 \%$ du strontium et environ $70 \%$ du ruthénium des légumes feuilles et gousses. Ces pourcentages ne changent guère pour une culture de légumes feuilles contaminée au stade plant ou au semis, alors qu'ils diminuent jusqu'à environ $30 \%$ pour les légumes fruits, pour lesquels la part de la translocation dans le transfert aux parties comestibles augmente avec le délai entre la contamination et la récolte. Dans le cas de légumes racines, l'épluchage est un moyen efficace d'éliminer $90 \%$ à $50 \%$ de l'activité.

Les résultats obtenus dans ce type d'expérimentations permettent de fixer un ordre de grandeur, applicable l'année culturale qui suit le dépôt, pour le cas d'une contamination atmosphérique accidentelle.

Dans le cas d'un transfert racinaire, la forme physico-chimique initiale des radionucléides n'a pas d'influence très marquée sur l'effet des procédés appliqués aux légumes récoltés, le sol jouant alors un rôle de tampon vis-à-vis des éléments incorporés au sol. Le parage est de loin le procédé le plus efficace pour éliminer les radionucléides pour les légumes racines avec l'élimination de l'ordre de $60 \%$ à $80 \%$ de l'activité. A l'exception du césium pour les légumes feuilles, éliminé à $90 \%$ par lavage et blanchiment, ces derniers traitements s'accompagnent d'une diminution de radioactivité de l'ordre de $15 \%$. La mise en conserve de légumes (précédée de parages, lavages, blanchiments) aboutit au total à une diminution de radioactivité de l'ordre de $90 \%$ pour les légumes racines et de $40 \%$ pour les gousses. Enfin, l'efficacité des traitements industriels des céréales est confirmée, les activités résiduelles des produits commercialisés étant au maximum de $50 \%$ de l'activité des grains récoltés. 
Ces résultats sont utilisables pour le cas de productions contaminées par transfert racinaire. Dans le cas d'un rejet accidentel, ils s'appliqueraient à la période de long terme après le dépôt, après une rotation culturale.

L'efficacité de ces traitements pour réduire l'activité des aliments peut être accrue, du fait de la décroissance physique, pour les radionucléides à courte période, car certaines transformations augmentent les possibilités de stockage des végétaux. En définitive, les processus étudiés s'intègrent dans l'ensemble des contre-mesures envisageables en situation post-accidentelle.

Les expérimentations ont été réalisées avec la collaboration technique de M. Hamoniaux et J. Marquet.

Les travaux présentés ont été partiellement soutenus par la Commission des Communautés Européennes (C.C.E. DG XII), par Electricité de France et par EURATOM, dans de cadre d'accords de coopération scientifique.

\section{RÉFÉRENCES}

[1] APOSTOLATO G., HADJANTONIOU A. - Caesium contamination transfer in wheat products. In : Seminar on radioactivity transfer during food processing and culinary preparation, Cadarache, 18-21 Septembre 1989. Cadarache : IPSN/SERE, 1989, 377-400.

[2] BRADLEY E.J., POPPLEWELL D.S., WILKINGS B.T. - The influence of simple culinary preparation on the radionuclide content of vegetables. In : Seminar on radioactivity transfer during food processing and culinary preparation, Cadarache, 18-21 Septembre 1989. Cadarache : IPSN/SERE, 1989, 311-324.

[3] CAWSE P., BAKER S.J., GRANDISON A.S., LEWIS M.J., PATEL S. - The influence of precessing on the radionuclide content of foodstuffs. In : CEC seminar on Intervention levels and countermeasures for nuclear accident, Cadarache, 7-11 Octobre 1991. Luxembourg : Commission des Communautés européennes, 1992, 271-288.

[4] LOFTI M., NOTARDO M., AZIMI-GARAKANI D., CUBADDA R., SANTARONI G.P., TOMMASINO L. - Concentrations of radiocaesium and its products after the Chernobyl accident. J. Environ. Radioact., 1990, 11, 177-182.

[5] VOIGT G., MULLER H., PROL G., PARETZKE H.G. - Caesium activity distribution in cereals after milling processes. In : Seminar on radioactivity tranfer during food processing and culinary preparation, Cadarache, 18-21 Septembre 1989. Cadarache : IPSN/SERE, 1989, 351-360.

[6] WEINER C. - Etude de polymères pour stabiliser des broyats de végétaux. Cas d'un polymère naturel : le scléroglucane. D.E.A. Interface chimie-biologie, Université de Montpellier I, Juillet 1992. 